\title{
Reduced right and left ventricular myocardial perfusion reserve in patients with scleroderma-associated and idiopathic (IPAH) pulmonary arterial hypertension due to impaired microvascular vasoreactivity
}

\author{
Jan Skrok*1, Monda Shehata1, Jie Zheng2 , Joao Lima ${ }^{3}$, David Bluemke , \\ Paul Hassoun ${ }^{5}$ and Jens Vogel-Claussen
}

\begin{abstract}
Address: ${ }^{1}$ Department of Radiology, Johns Hopkins University School of Medicine, Baltimore, , USA, ${ }^{2}$ Mallinckrodt Institute of Radiology, Washington University School of Medicine, Saint Louis, MO, USA, ${ }^{3}$ Department of Cardiology, Johns Hopkins University School of Medicine, Baltimore, USA, ${ }^{4}$ Department of Radiology and Imaging Sciences, National Institutes of Health, Bethesda, USA and ${ }^{5}$ Department of Pulmonology, Johns Hopkins University School of Medicine, Baltimore, USA

* Corresponding author
\end{abstract}

from I3th Annual SCMR Scientific Sessions

Phoenix, AZ, USA. 21-24 January 2010

Published: 21 January 2010

Journal of Cardiovascular Magnetic Resonance 20 I0, I 2(SuppI I):P258 doi:I0.I I86/I532-429X-I 2-SI-P258

This abstract is available from: http://jcmr-online.com/content//2/SI/P258

(C) 2010 Skrok et al; licensee BioMed Central Ltd.

\section{Introduction}

In patients with pulmonary arterial hypertension (PAH), the right ventricle (RV) undergoes hypertrophy and remodeling, eventually leading to RV failure and death. Patients with left ventricular (LV) failure are known to have reduced myocardial blood flow (MBF). The relationship of LV and RV myocardial perfusion reserve (MPR) in patients with PAH remains unclear.

\section{Purpose}

To evaluate biventricular perfusion at rest and under adenosine stress in patients with known or suspected PAH in relation to biventricular function and pulmonary hemodynamic parameters.

\section{Methods}

Twenty-five patients (59.6 years, 52.8-66.6, 22 females) who were referred for known or suspected PAH underwent adenosine stress cardiac MRI and right heart catheterization (RHC) on the same day. They were subdivided into groups with PAH ( $\mathrm{mPAP}>25 \mathrm{mmHg}, \mathrm{n}=17,11$ scleroderma-associated, $6 \mathrm{IPAH})$ and without PAH (mPAP < $=25 \mathrm{mmHg}, \mathrm{n}=8$ ). First-pass perfusion GRE images were obtained at 3 T (Magnetom Trio, Siemens, Germany), using gadopentetate dimeglumine $(0.025 \mathrm{mmol} / \mathrm{kg})$ as a contrast agent. Biventricular global MBF was calculated by MBF mapping, using the Fermi function model (Figure 1). Individual groups were compared using the Mann-Whitney-U test; correlations between MPR and biventricular function parameters were tested using the non-parametric Spearman's rho correlation coefficient.

\section{Results}

RV MPR in the PAH group (1.7, 1.3-2.0) was significantly lower compared to the non-PAH group $(2.5,1.8-3.9 ; \mathrm{p}=$ $0.03)$. LV MPR in the PAH group $(1.8,1.6-2.1)$ was also significantly lower compared to the non-PAH group (4.1, 2.6-4.8; $\mathrm{p}=0.0003)$. There was a significant correlation between RV and LV MPRs $(\mathrm{r}=0.62, \mathrm{p}=0.0008)$. There was a trend towards higher resting and lower stress myocardial blood flow in the PAH group compared to the non-PAH group for both ventricles.

There were significant correlations between LV MPR and $\operatorname{mPAP}(\mathrm{r}=-0.79, \mathrm{p}<0.0001)$, PVRI $(\mathrm{r}=-0.72, \mathrm{p}<0.0001)$, PCWP $(\mathrm{r}=-0.45, \mathrm{p}=0.03)$, and RV function parameters (RVED volume/BSA $(\mathrm{r}=-0.42, \mathrm{p}=0.04)$, RVESvolume/ BSA $(\mathrm{r}=-0.48, \mathrm{p}=0.01)$, RVEF $(\mathrm{r}=0.53, \mathrm{p}=0.007)$, RVmass/BSA $(\mathrm{r}=-0.53, \mathrm{p}=0.006)$, RV septomarginal 


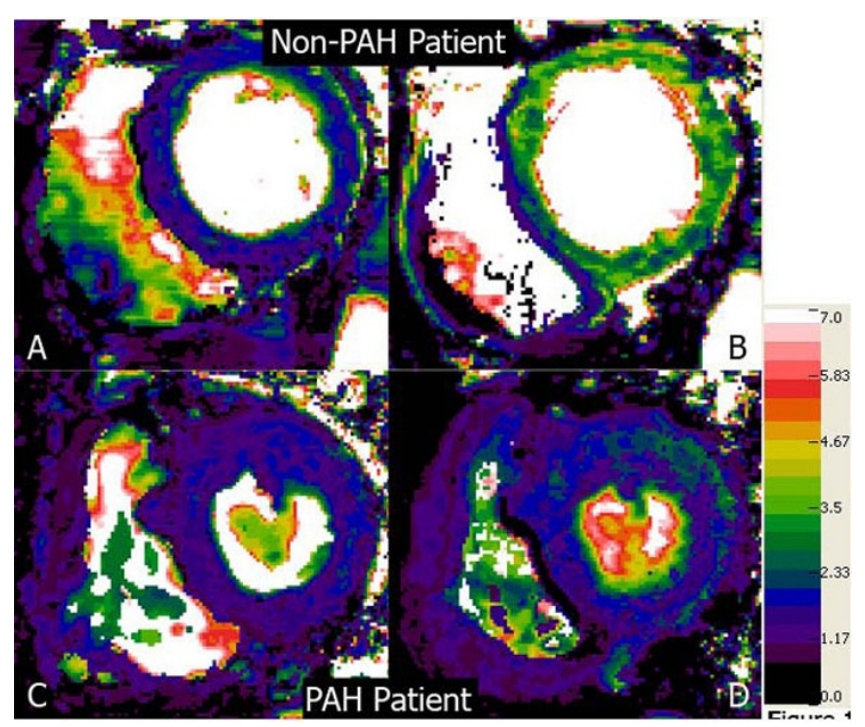

Figure I

A, B: Myocardial blood flow (MBF) images in a nonPAH patient (58 year-old female, mPAP I $2 \mathrm{mmHg}$ ) demonstrate a marked increase from rest $(A)$ to adenosine-induced stress (B) conditions. C, D: In a patient with scleroderma-association PAH (67 year-old female, MPAP $20 \mathrm{mmHg}$ ), there is only a mild increase in biventricular MBF from rest (C) to stress (D).

trabeculation (SMT) mass/BSA $(r=-0.63, \mathrm{p}=0.0007)$ and ventricular mass index $(\mathrm{VMI})(\mathrm{r}=-0.54, \mathrm{p}=0.007)$.

For the RV, there were significant correlations of the MPR with $\operatorname{mPAP}(\mathrm{r}=-0.59, \mathrm{p}=0.002)$, PVRI $(\mathrm{r}=-0.48, \mathrm{p}=$ $0.01)$, RVESvolume/BSA $(\mathrm{r}=-0.44, \mathrm{p}=0.03)$ and RVEF $(\mathrm{r}$ $=0.47, \mathrm{p}=0.02$ ).

There were no significant correlations with LV function parameters, Figure 1.

\section{Conclusion}

Reduction of not only RV but also LV MPR in patients with IPAH and scleroderma-associated PAH can be explained by microvascular/endothelial dysfunction affecting both ventricles. The biventricular vasoreactivity in response to adenosine correlates with the severity of pulmonary pressures as well as RV failure and RV remodeling.

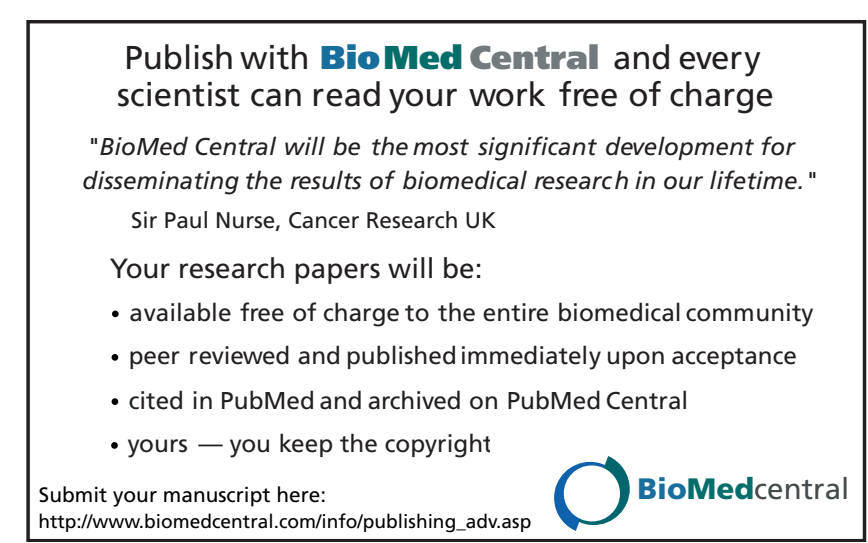

